for outlying communities? The suggestion has been offered that the best mode of solving this serious problem lies in the establishment by communities-by counties or groups of counties-of public hospitals, offering all the conveniences needed for first-class practice. And the argument has been put forth that such a hospital in a center from which radiated a system of good roads could provide medical care for a wide area. Further, because of the common use of the hospital, physicians would be frequently brought together in consultation and conference, with the result that professional discouragement and decay resulting from isolation would be remedied and the young practitioner would be attracted. Again the solution which has been offered may not be the best one and on further examination may be shown to have grave defects. And even if it were regarded as the most satisfactory way out of the crisis which seems to be approaching in rural life, there would remain the necessity of persuading people of its desirability and of the reasonableness of spending public money for hospital construction and upkeep.

Such, then, are some of the puzzling questions which are of concern not only to the medical profession but to the public at large at this time. We, as servants of the common weal, should become keenly conscious of them and give our best thought to answering them. Attention to these questions will take us out of the routine of our daily duties, and bring us into relation to other's who as public-spirited citizens are looking forward to and working for a better day. With them we must think out as clearly as possible the conditions which will be best for the welfare of all people and then strive to establish them. The opportunity for both professional and public service is a great one for us all, but especially for those who are just now entering on the duties of physicians.

\section{(Briginal Artixlax.}

\section{THE FAILING HEART.*}

BY PAUL D. WIITTE, M.D., BOSTON.

INTRODUCTION.

HEART FAILURE consists of the inability of the heart to carry on a circulation sufficient for the ordinary life of an individual. It is thus a relative term. The amount of work routinely performed by one man may suffice to produce heart failure in another man who does not show anv cardiac weakness in the habitual pursuits of his life. Mackenzie defines heart failure as "the condition in which the heart is unable to maintain an efficient circulation when called upon to meet the efforts necessary to the daily life of the individual." 1022 .
The term "cardiac insufficiency", is, on occasion, preferable to "cardiac failure," for when the latter expression is used the average man in the community at once thinks of failure in its extreme phase when the victim is about to depart this life. "Decompensation" is an unsatisfactory term which I for one would discard with pleasure.

In general, there has been too much mystery about heart failure-in the books too much about back pressure, reserve force, restored compensation and all. The complication of the discussions of the subject has clouded it. There is still something to be learned about heart fail. ure, but what is known may be, I believe, more clearly expressed than often one finds it.

How is the circulation normally maintained? By the proper action of the heart muscle and coöperation of arteries and capillaries. That circulatory insufficiency which is the result of failure of the peripheral circulation, and not of heart failure, I shall not dicuss, except clearly to point out that these two causes-cardiac and arterial-of circulatory failure must be differentiated. Examples of peripheral circulatory failure are found in ordinary syncope, so-called surgical or traumatic shock, endarteritis and thrombosis.

TYPES OF IIEAR'T FAILURE.

Lewis has differentiated two types of heart failure or cardiac insufficiency, one in which dyspnea and congestion are the chief features, and the other in which pain, severe or mild, in the region of the heart, is induced by exertion. The former he has called "failure of the congestive type," and the latter "failure of the anginal type." Sometimes both pain and congestive symptoms and signs occur together, but more frequently they seem to be separate. It should be emphasized that angina pectoris or heart pain may be just as important evidence of cardiac insufficiency as edema; in fact, often much more so, if severe. The trem "pseudoangina" is a poor one and should be dropped. Many of the cases showing it prove later to have the so-called "true angina pectoris," dependent on actual sclerosis of the coronary arteries, and those that do not can be better classified, as I shall show shortly. There may, of coursa, be all graduations of both types of heart failure.

CAUSES OF IIEART FAILURE OF CONGESTIVE TYPE.

I shall discuss now both types of cardiac failure or insufficiency from the standpoint of cause, diagnosis and treatment. First let me take up the "congestive" type. The etiology for heart failure is fundamentally the same as that for heart disease plus the necessary overexertion or excitement which acts as the last straw to break the camel's back. The common causes of heart disease are, therefore, the common basic causes of heart failure. Arteriosclerosis, rheumatism. chronic hypertension, syphilis and thyroid intoxication are in about 
that order the important factors. Sometimes two or three of the causes are combined in an individual case. But in the study of a patient with symptoms or signs of eardiac insufficiency it is not enough to make a diagnosis of heart failure; it is just as important to analyze and state the funrlamental eause if it can br found. and if it cannot be found, it should be so declared. Also structural changes in valves and heart chambers should be ascertained and diagnoseci, for they do make a difference in prognosis and treatment. The pendulum has begun to swing over from the former complacent attitude of the medical profession in the diagnosis of mitral regurgitation and cardiac dilatation towards simple expressions of functional capacity, but it tends to swing too far. The stethoscope is still a useful instrument, as are also the blood pressure cuff, the x-ray and the electrocardiograph. We must simply not read into their findings undue significance. In the presence of heart failure I like to know, for example, whether the patient is one showing also rheumatic heart disease, mitral stenosis and auricular filcrillation, or syphilitic heart disease with aortic regurgitation and normal rhythm. Prognosis and often treatment will vary according to etiology.

\section{DIAGNOSIS OF CONGESTIVE FAILURE.}

The first evidence of the onsct of congestive failure is dyspnea on some ordinary exertion. From this symptom a steady progression to edema, cough and cyanosis may occur and of course when these develop the diagnosis becomes obvious. With dyspnea alone, however, diagnosis is not so simple. Probably just as frequently, or even more so, dyspnea is a symptom of nervous fatigue as expressed by the so-called effort syndrome. In young people this condition of exhaustion of an oversensitive nervous condition is very common. We found it everywhere in the army and I find it everywhere in eivil life. Girls labelled "mitral stenosis" are as likely to have "effort syndrome" as mitral stenosis; young chaps labelled "gassed in France" are much more likely to have "effort syndrome" than any effect of the gas on their hearts; in fact, almost certainly so. This is a very vital matter to recognize, for the whole future of the individual may depend on the early and proper realization of the condition. I have seen a girl of about 20 who had been kept as an invalid for several years with the warning never to marry who had just as normal a heart as yours or mine, and perhaps more normal. She was of nervous temnerament, showed dysnnea on exertion and had a sharp first sound which had been wrongly called a presvstolic murmur. It required much explanation on my part to convinee her that she was just, high strung but normal as to her heart after the hermit's life she had lived for years. Very helpful points in distingnishing between dyspnea of heart disease and of effort syndrome are that there is apt to be more palpitation with effort syndrome and that there is often actually more heart pain of one kind or another. Very helpful additional evidence against heart disease as the cause of the shortness of breath is the story in young people of dizziness with or without actual syncope. If a young girl comes to me, complaining of fainting, palpitation and shortness of breath, I should be right ninety-five times out of a hundred if I considered her a case of effort syndrome rather than of heart disease even before I examined her. The term "effort syndrome" is weak in that it does not express the effect of excitement on such hearts, often much more striking an effect than that from effort. Neuro-circulatory asthenia was a term used in this country for the condition during the war. Nervous heart is another term in use.

I should like to make one further observation about this very important condition of effort syndrome, and that is, that it may coexist with heart disease, especially in young people. I find almost invariably that the symptoms in such people are ascribed by the doctor to the heart trouble, when as a rule a considerable percentage, and sometimes even a great preponderance, of the symptoms are the result of the associated condition of effort syndrome.

Other less common conditions than effort syndrome, to be differentiated from heart failure, are hyperthyroidism and subacute or chronic infections, which after all really express themselves cardiovascularly as effort syndrome. 'Thuberculosis, subacute endocarditis, and lowgrade septicemias are particularly apt to be mistaken for slight heart failure. Anemia, primary or secondary, and malignant disease are other conditions, and chronic nephritis still another, if producing toxic symptoms, to be distinguished from a failing heart. Chronic bronchitis, hepatic cirrhosis and phlebitis with edema may rarely simulate cardiac insufficiency. A recurrent acute endocarditis in an old rheumatic heart is particularly apt to be confused with heart failure. Careful temperature records and blood counts are often necessary.

What about asymmetrical failure of the ventricles? And how does the old back-pressure theory fit into it? There is little question but that in chronic mitral stenosis, where the right ventricle has hypertrophied preponderantly to maintain the much-increased pressure of the nulmonary circulation, the strain may finally become too great for it. It may begin to fail before the left ventriele does. This is not back pressure. It is just inability of the right ventricle to maintain a proner forward pressure. The lungs become more and more engorged and edematous, and the hloof nonrer and poorer in oxvegen. This affects the whole body and, incidentally, through the enronaries the left ventricle, which eventually also begins to fail. Then general edema appears in the legs and belly, and finally 
we have a picture of extreme failure of the whole heart. It is similarly conceivable that in chronic systemic hypertension (as opposed to pulmonary jypertension) the left ventricle. which carries. the burden, may be overstrained and begin to fail. Edema appears, coronary circulation is interfered with, and so the vicious circle goes on with the right ventricle finally failing also because it is poorly supplied with blood. It appear's to me that this explanation of asynchronous onset of ventricular failure seen in some cases is a reasonable one; it is supported by a good deal of evidence.

Whether or not it is possible to tell which coronary is thrombosed by evidence of the earlier failure of one ventricle than the other I do not know. At the present time I have under my care a man who a month or more ago had probable coronairy thrombosis (evidenced by very severe prolonged pain requiring repeated doses of morphia and followed by acute pericarditis and paroxysmal auricular fibrillation and great weakening of the first sound, with an old history of repeated brief attacks of angina pectoris). He now has pulmonary edema with hemoptysis and cyanosis with little or no edema elsewhere. He may have thrombosis of his right coronary artery, or of that part of the left coronary which helps to supply the right ventricle.

\section{TRIATMENT OF CONGESTIYE FAIL.URE.}

Now as to the treatment of heart failure of the congestive type. One of the very commonest conditions associated with this type of failureand indeed in large part responsible for it-is auricular fibrillation or absolute arrhythmia of the pulse. When a patient with this arrhythmia is first seen, before treatment, the apical pulse rate is usually very high, averaging perhaps 150 per minute and fitting in every way the old term delirium cordis. In such a patient prompt and proper treatment results in such vast and striking benefit that its recognition and relief constitute one of the demonstrations of the real value of the doctor in the community. I dare say medical reputations in practice are not infrequently established by the fact that foxglove by its poisonous effect on the junction of the auricular muscle and the node of Tawara acts as a sedative to the heart. Thus only indirectly is digitalis a stimulant - by far its greatest benefit comes from its poisonous sedative action. And indeed it is worth while to recognize auricular fibrillation as an essential factor in the production of much heart failure of the congestive type. The failure results from the strain of a very rapid heart beat on a diseased or weak or exhausted nyocardium. The speed is more important, I believe, than the rhythm; for once the rate is reduced circulation is restored in most cases in spite of the persistence of the irregularity. Simple as this may seem, the discovery in actual practice of improper treatment of the failing heart with auricular fibrillation makes me feel that too much emphasis cannot be placed upon it. Usually too little digitalis is. given or it is given too slowly. As one authority has said, many people have died as the result of digitalis therapy, because too little was administered At times perhaps the actual quantity of dried leaf or tincture or infusion may have seemed sufficient, lut a feeble leaf may be responsible for its inertness. However, I think it may be truthfully said that nowadays most of the digitalis preparations on the market are of good potency.

An example of the treatment of a patient with heart failure of the congestive type may interest you. It was to me a striking lesson. A woman of 55 years, seemingly on her death bed, I saw in consultation in an outlying town eighteen months ago. She was very ill, orthopneic, cyanotic and markedly edematous. She was. raising bloody sputum. Her apex pulse rate was. 164, absolutely irregular; her radial pulse 142, a pulse deficit therefore of 22 . She had had" rheunitic fever in childhood and for a year past had had palpitation, dyspnea and precordial distress, apparently somewhat relieved by small amounts of digitalis. She had become acutely worse two weeks before I saw her. Small amounts of digitalis having failed to help her, she was put on crataegus oxyacantha-5 drops. every three hours, with 1/100 grain of strychnine and 5 drops of whiskey every half-hour. She was losing ground fast and it did not seem. that she could live overnight. I found mitral' stenosis and auricular fibrillation present, undonbtecily of rheumatic srigin. She promised to be a farorable case for saturation with digitalis and T gave a hopeful prognosis. Her heart was obviously erying for digitalis (as showr. particularly by the very rapid, irregular apex rate). We therefore began giving her digitalis leaf by mouth (and, by the way, it is very rarely necessary, even in such an emergency as this case was, to give digitalis by vein or under the skin.) Three grains (0.2 gram) were given every four hours and the order was left to continue this dosage until the apex rate fell below 80 or until the patient was toxic (as shown by nausea or a coupled pulse). All other drugs were omitted.

The next day she was already better, and when I telephoned on the second morning I found she was wonderfully improved, the apical pulse rate 68 without deficit, but still irregular, of course, the edema vanishing like snow in the spring. sun and the cough stopped. She could lie flat with eornfort. This mirneulous effret wits accomplished, I am convinced, by the reduction in the pulse rate. The heart was beating 96 times less often every minute than it had been beating two days before. Just think what this means to a tired heart--in 24 hours, 138,000 contractions cut out!! No wonder the myocardium was recovering and the circulation restored. Shortly this patient was up and around, and within the 
past month I have heard from her doctor that she is in very fair health.

In connection with this case I want to emphasize the importance of recording the apex pulse rate-it should always be done when auricular fibrillation is present. Both apex and radial pulse rates should be taken simultaneously by two observers, or else in successive minutes by one observer and the difference graphically represented, if possible; the difference is the pulse deficit. The reduction or melting away of this deficit under digitalis therapy is a good guide in treatment. Yet often one finds even in unexpected places that the radial pulse is the only pulse record on the nurse's chart. I. remember one striking case in which, according to the chart, which I examined first before I listened to the heart, the pulse was recorded as 90-odd, feeble and irregular. In this case the apex rate was over 170 and auricular fibrillation was present. The institution in this patient of proper treatment, with relief, actually depended on the recognition of this fact."

I do not believe in overdoing the matterthat is, in giving too big a dose of digitalis at the outset. In the first place, one may not be absolutely certain about possible digitalis already taken, and secondly, there is no particular point in making the patient nauseated, even though in the process the heart is vastly improved. Also rarely digitalis kills. Intense nausea and vomiting and the bigeminal pulse are indications that we are close to the danger point in digitalization-probably nearly 90 per cent. of the lethal dose has been given. There is a happy medium between the small doses of bygone days and the massive doses which we have recently tried in some cases. The size of the individual must be taken into consideration, particularly in the estimation of a proper dosage. Luast night I saw a woman weighing only a few pounds over 80 , who, having needed digitalis badly, had been given 3 grains ( 0.2 gram) of the leaf (a potent preparation in this case) three times a day. After two days she became intensely nauseated and had very severe gastro-intestinal symptoms for over 24 hours, with almost constant vomiting. When I saw her she was already getting over the worst: effects of the drug and looked quite well, b:at was still nauseated. She had rheumatic heart disease, mitral stenosis and auricular fibrillation. Her apex rate had fallen from 130 to 58, and her circulation was almost completely restored. IJowever, a person of her weight should have received a smaller total dose and she would have been better off. 'This illustrates the opposite error in the administration of digitalis.

One other case I want to cite here-a woman who entered the wards of the Massachusetts General Hospital 18 years ago. She had rheumatic heart disease and auricular fibrillation.

*Mackenzie feels that too much emphasis should not be placed on the "pulse defoit" since the figure for the radial pulse rate in auricular fibrillation varies with the ability of the observer to count the beats at the wrist.
I have looked up her record and I find that she was treated for weeks and given tincture of digitalis in insufficient quantity and only at intervals. She actually was discharged from the hospital with edema still present and a high apical pulse rate (over 110), simply because the physicians in one of the best hospitals in the country, even as recently as this, did not know how to give digitalis and did not recognize the significance of auricular fibrillation. Under a very discerning clinician outside the hospital this patient improved and lived for years.

I cannot leave the very important subject of the treatment of heart failure of the congestive type without four more brief observations. First, it is essential that, after bringing the heart of a patient with auricular fibrillation under the control of digitalis, it be maintained under that control. Again, this sounds simple, but it is everywhere neglected. After a striking recovery under digitalis the patient with auricular fibrillation, whether arteriosclerotic or rheumatic in origin, goes on his way rejoicing. All goes well for a few weeks and then the pulse slowly climbs again, and with it comes another "break" in circulation. The process is repeated time after time; much work and money are sacrificed by the victim and undoubtedly his life is shortened. The rational procedure is to maintain digitalization in a patient once after securing it if the patient really needs the drug. Of course, this applies particularly to cases with auricular fibrillation. Give daily about the amount which is excreted. This is about $11 / 2$ grains (or 0.1 gram) of the leaf in the average adult, who has been digitalized by about 22 grains (1.5 grams) in divided doses in two or three days. By maintaining this digitalization a patient may keep at work for years and undoubtedly live longer. It does no harm, so far as anyone knows, to take digitalis daily for years. Withering, more than a hundred years ago, knew more about the proper use of digitalis than many physicians do today.

The second point concerns the administration of digitalis to patients with normal rhythm. 'There is experimental evidence, and clinical, too, that even when the pulse is regular and fairly slow digitalis does help in failure of the congestive type. Apparently, in addition to its toxic effect to block off stimuli from auricles to ventricles, thereby resting the heart, there is also a direct stimulating action on the ventricular muscle. Such cases of congestive failure respond much less dramatically, however, to digitalis than do patients with auricular fibrillation.

The third point concerns the manner of giving digitalis and the use of other digitalis-like drugs in congestive failure. I have found that a good leaf in pill form by mouth is the most satisfactory therapeutic method. There is no virtue of the tincture or of the infusion or of various costly pharmaceutical preparations of 
digilalis over the leaf itself, provided one knows that the leaf is potent. In hospital use the ordinary leaf is one-tenth to one-fortieth als expensive as various tracle preparations, and apparently just as effective, Other drugs of the cligitalis series-strophanthus, supill, apocymm and convallaria-may be dismissed with the simple statement that for heart failure of the congestive type they are inferior-all of them-to digitalis. (Other drugs, such as stryehnime, campliol, caffeine, alrenalin, cactus and cratacous, are probably of no value as cardiac stimulants. To be sure, caffeine is a good respiratory and nervous stimulant and will at times break up periodic breathing such as th Cheyne-Stokes, restoring a regular respiration; adrenalin will for the moment raise the pressure and dispel the circulatory failure of shock; and strychnine is a stimulant for the central nervous system; but there is little or no evidence that they affect the heart, and until there is I believe we should use such drugs only symptomatically-some of them, such as cactus, not at all. Quinidine is not a drug for the treatment of lieart failure. Its sole function is to stop or prevent auricular fibrillation in selected cases.

I should like to digress here for a moment to say that I do not believe in giving a multitude of stimulants (so called) in infectious disease to "prevent the heart from failing." One of the most successful recoveries I have ever witnessed from severe exanthematic typhus was in a man who liad two excellent nurses but no dirigs at all.

Finally, the fourth point $I$ want to make briefly is that besides the use of digitalis in congestive failure we have numerous other important measures. These are simple and widely followed, and I shall merely name them and then pass on to the final part of my talk: absolute rest in bed while there is failure; morphia if there is pain or great discomfort; veronal or bromides if sleeplessness; diuretics (caffein, diuretin, theocin, calomel) if there is obstinate edema not vanishing with rest in bed and digitalis; reduction in quantity and richness of food (sometimes the extreme limitation of the Karell diet is helpful) ; catharsis (but not enough to exhaust the patient) and venesection in rare cases.

DIAGNOSIS OF HEART FAILURE OF ANGINAL TYPE.

And now I shall discuss briefly the very important heart failure of the anginal type. Here the only evidence of any sort may be the heart pain. There may be no other symptoms and there may be absolutely no signs of heart disease, and yet serious myocardial disease may be present. Mackenzie has recently told the story of a man of 60-odd who came to see him some six years ago because of heart pain. Although he could find no abnormality of the heart or circulation on physical examination, he diagnosed coronary selerosis and warned the patient. Ile did not see this man again until two years later, when the patient visited him once more and said that he was worse. He admitied that he had not heeded Mackenrie's warning, but had continmed an active life. When asked why, he said that at the request of friends he had visited a prominent doctor in a far (e)imtry. There many specialists had examined him and tested the contents of his stomach. bowels, bladder and veins; had taken x-rays and electrocardiograms, had determined his metabolism, and had tested the effect of various diets. All the results were negative and the man was pronounced normal. Because there was no instrument to measure and record the pain it was ignored. The man died later with angina pectoris. 'This is an extreme case, but it does support Mackenzie's contention that as aceurate as seem to be oul instruments of precision, they cannot reveal everything-they are still not infrequently too erude to show the more delicate pathological changes in the human bodly. Sometines we are fortumate in the study of a doubtful case of angina in finding evidence. of cardiac enlargement or aortic dilatation by $\mathrm{x}-\mathrm{r}$ ay, the extremely significant intraventricular heart block by electrocardiogram, the weak first heart sound at the apex by stethoscope or a positive Wassermann reaction. Oceasionally all these findings are absent and yet the patient may dic suddenly and show at autopsy coronary selerosis, or even, in the severer cases, coronary thrombosis, with perhaps hemorrhage into the pericardium through a tear in a waikened infareted ventricular wall.

Age at or beyond middle life and heart pain of almost any degree are the essential factors in the diagnosis. 'There may or may not be cardiac enlargement or high blood pressure or murmurs or poor sounds or pulsus alternans or peripheral arteriosclerosis (such as radial sclerosis or arcus senilis). Young people with heart pain almosi always have the umimportant. effort syndrome and must be differentiated. Such cases usually show also dyspmea, palpitation and.nervousness. In these youths with effort syndrome sometimes the heart pain may be very severe and radiate even down the arms. As a rule, in an older person the pain is the essential symptom; it comes on as the result of exertion or excitement or the pressure of a full meal, before there is time for the development of dyspnea.

Acute or chronic abdominal disease (especially gallstones) often has to be differentiated from angina pectoris; rarely pleurisy or pericarditis may simulate it. Many attacks of "acute indigestion" in old people are simply angina pectoris.

There is rarely, if ever, edema except in the late stages, when, after years of angina, the congestive type of failure may supervene. The patient, however, rarely lives long enough to ilevelop the congestive type of failure, but I 
have seen recently a doctor, 70 years old, wlio began to have definite angina pectoris 12 years ago and who is now showing dyspnea and edema. As this case indicates, the condition is by no means immediately fatal, or even fatal in a few years, in all patients. It is one of the most difficult conditions in the practice of medicine to which to attach a reasonably accurate prognosis. An extremely rare combination of cardiac conditions is due to the coincidence of chronic auricular fibrillation and angina pectoris. This has been clearly recognized by Mackenzie and others. I have seen but one such case in arteriosclerotic heart disease. Paroxysmal auricular fibrillation with coronary thrombosis is not so rare.

\section{OAUSE OF HEART FAILURE OF ANGINAL TYPE.}

The cause of failure of the anginal type seems to be a malnutrition of the myocardium as the result of coronary sclerosis. Ordinary arteriosclerosis is the essential etiological factor, but syphilis plays an undoubted part in some cases. I do not believe that the condition is always the result of aortic changes (chiefly an aortitis), as suggested by Allbutt. I feel that myocardial irritation and disease as the result of temporary or permanent insufficient blood supply, because of abnormal coronary arteries, is a more important factor. The site of origin of the pain is interesting; it is often substernal, but also sometimes apical. Perhaps this variation is dependent on the site of the chief lesions -aortic or coronary. It seems probable that the angina of luetic aortitis and of coronary arteriosclerosis may be of different nature. This needs further study and analysis, and I have not had time tonight to go into the details of the variations in position and intensity of angina pectoris. It may be said, however, that the pain of true angina inay be substernal or apical (more often the former), gripping or knife-like, constant or transitory. Interesting variations may occur, such as epigastric pressure, right chest ache, or numbness of the left wrist on exertion actually antedating substernal pain in the same case.

The mechanism of the production of the pain is not yet clear. It seems possible that a constriction of irritable coronaries may account for the usual attaoks of angina, while for the prolonged terrible heart pain coronary thrombosis is responsible.* It is conceivable that coronary branches of various sizes may be irritable or thrombosed, giving rise to all gradations of symptoms and signs. When one says that coronary thrombosis is always fatal in minutes,

*It is my belief that heart pain is of flve types-first, the socalled true angina pectoris due to coronary arterial disease and constriction with resulting myocardial anemia and exhaustion, re lieved by nitroglycerine; second, aortic pain with aortitis; third right ventricular fatigue as in chronic mitral stenosis, the pain being apical in location and not relieved by nitroglycerine but often helped by rest and digitalis; fourth, left ventricular fatigue as in chronic systemic hypertension or aortic stenosis, helped mor by digitalis and rest than by nitroglycerine; and fifth, the pain in effort syndrome probably resulting from overactive heart contractions in a person with low nervous threshold. The writer continuing a study of an analysis of heart pain. hours, days or weeks, one means that if a large enough artery is thrombosed the patient will die. I have secn at least temporary recoveries in several cases with probable coronary thrombosis.

Louis Gross of Montreal, in an excellent book on the coronary circulation (which it would benefit you all to read), has demonstrated the remarkably extensive coronary anastomoses in the human heart, particularly with advancing age, through the establishment of new vessels to supply the pericardial fat. He incidentally cites the case of an old woman who died of malignant disease. At autopsy it was found that her right coronary artery was completely thrombosed. Her myocardium was, however, almost perfectly normal as the result of anastomoses through the left coronary artery. She had in life never shown any symptoms or signs of heart trouble.

One further comment should be made on the subject of anginal failure before I end this talk with a brief discussion of its treatment. Nervous, high-strung people are probably more likely to show angina than placid individuals with the same degree of arterial damage. Their blood pressure, of the so-called essential hypertension type without nephritis, is apt to bob up and down readily at the least provocation. At such times of provocation their angina is apt to appear. I think this point must be borne in mind in prognosis-i.e., such people, if treated properly, may do fairly well for some time to come.

\section{TREATMENT OF ANGINAL FAILURE.}

Now as to treatment. Rest is essentialmental and dietetic, as well as physical; that and the relief of the pain. Nitroglycerine or amyl nitrite, the old stand-bys, are, of course, invaluable and should always be carried by the patient suffering from heart pain of the "anginal" type. Morphine should be given freely if necessary, and when much of it is necessary coronary thrombosis is probably the underlying condition. Digitalis is generally contraindicated in the anginal type of heart failure but may help a good deal if the heart pain is due to right ventricular fatigue in mitral stenosis or to the left ventricular fatigue in chronic hypertension. A light diet, particularly light in bulk, is of great importance; quantity is much more vital than quality. Five small meals a day, with the last one the lightest, are a good program. Avoidance of taxing business cares as well as absolute omission of abruptness in physical activity must be advised. Rest will not only cause the cessation often of anginal pains but incidentally is by far the best treatment for hypertension which may or may not be associated with the angina. I have found, however, as low a blood pressure as $115 \mathrm{~mm}$. systolic in marked coronary sclerosis. If syphilis is proved or likely, antiluetic treatment, begun with care, may be very beneficial. 
SUMMARY

1. There are two general types of failing hearts-the congestive type and the anginal type.

2. All the causes of heart disease are likewise responsible for heart failure of the con gestive type. The commonest causes are arteriosclerosis, rheumatic fever and allied conditions, chronic hypertension, syphilis and chronic hyperthyroidism.

3. A common and very vital factor in the immediate causation of heart failure of the congestive type is auricular fibrillation. Its recognition and proper treatment by digitalis is veyy important. Overexertion in normal rhythm may also cause failure.

4. Heart failure of the congestive type must be differentiated from effort syndrome, hype.thyroidism, acute infections, pulmonary disease and anemia.

5. Heart failure of the anginal type is the result . of coronary sclerosis in general, with syphilis sometimes a factor. In this type auricular fibrillation is uncommon, except occasionally in paroxysms.

6. Anginal failure must be differentiated from the heart pain of mitral stenosis, of hypertension and of effort syndrome, and from acute and chronic abdominal disease.

7. Anginal failure may be present in a person with no signs of heart disease and no other symptoms.

8. Digitalis is. as a rule, not indicated in anginal failure. Nitroglycerine or amyl nitrite, morphia, but chiefly rest--physical, mental and dietetic-are the therapeutic measures to be followed.

9. The amount of cardiac reserve in both types of heart failure is often amazing, the patient sometimes living for many years after the first symptoms of heart failure have appeared?

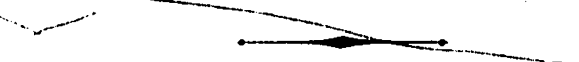

A PRELIMINARY REPORT ON THE TREATMENT OF CARCINOMA OF THE ESOPHAGUS WITH COLLOIDAL SELENIUM.

BY ELMER B. FREEMAN, M.D., BaLtiMORE.

\section{[From the Digestive Clinic of Johns Hopkins} Hospital.]

THE anatomical relations of the esophagus are such as to make the diagnosis of esophageal diseases very difficult and the treatment even more so. Also, when one considers the relations of the esophagus to the trachea and the thyroid in the neck, the left bronchus, the arch of the aorta and the heart in the chest, it becomes very apparent that the pathological conditions in these organs may, and frequently do, produce symptoms which are referred to the esophagus. It must also be borne in mind that diseased conditions quite remote from the esophagus may re- flexly produce esophageal symptoms. Therefore, before discussing the treatment of carcinoma of the esophagus with colloidal selenium, I desire to briefly mention some of the symptoms of esophageal diseases and the special methods of study employed in the differentiation of the same.

Usually the first manifestation of esophageal disease is dysphagia, but frequently a lesion may have existed for some time before this symptom appears. This is true especially in carcinoma, while in spasmodic disease dysphagia is frequently an early symptom. When one considers that the esophagus is subject to marked mechanical irritations from coarse, irritating foods, to thermic irritation from hot and cold liquids and also to chemical irritations, it seems rather remarkable that pathological lesions do not occur more frequently; also, when it is remembered that the capacity of the normal esophagus is only 100 c.c., that the lumen is quite small, that for comfort in the swallowing act we depend very much on the elasticity of the esophagus, that, if much narrowing occurs at any point, ob. structive symptoms occur promptly.

While dysphagia is the first symptom to appear in the majority of cases, it may sometimes be preceded by the sensation of fullness or discomfort or even by pain, that is dull and aching or sometimes acute and sticking in character, either in the sternal or epigastric regions. However, the slightest dysphagia or discomfort referred to the region of the esophagus should call for a very careful study, always bearing in mind that the symptoms referred to may be caused by pathological lesions quite remote from the esophagus. The dysphagia which is due to carcinoma is characterized in the beginning of the obstruction by difficulty in the swallowing of solid foods only, but after the disease progresses and the obstruction increases, even liquids are swallowed with great difficulty. Finally, complete obstruction occurs when nothing will pass through the esophagus.

On the contrary, spasmodic disease is characterized by a sudden onset and symptoms of almost complete obstruction which in a short time give way to normal swallowing, to be followed again by a return of obstructive symptoms. In these cases the obstructive symptoms are quite as pronounced with liquids as with solid foods. Associated symptoms depend mostly on the degree of dysphagia present. As long as the patient is able to take food in sufficient quantity, the condition remains quite satisfactory, but when they are no longer able to obtain sufficient nourishment, they begin to show definite signs of malnutrition and anemia and, in the carcinomatous cases, cachexia as well.

A routine physical examination gives us practically no information in regard to the esophagus, for one can palpate only a small portion of the esophagus above the thorax. This portion is so deeply located that even palpation is unsatisfactory. There is nothing to be made out 